\title{
Abundância sazonal de aves migratórias na Área de Proteção Ambiental de Piaçabuçu, Alagoas, Brasil
}

\author{
Susanna A. S. Cabral; Severino M. de Azevedo Júnior \& Maria Eduarda de Larrazábal
}

Departamento de Zoologia, Centro de Ciências Biológicas, Universidade Federal de Pernambuco. Avenida Professor Moraes Rego 1235, Cidade Universitária, 50670-420 Recife, Pernambuco, Brasil.

E-mail: sascabral@hotmail.com; smaj@ufpe.br; mells@ufpe.br

\begin{abstract}
Seasonal abundance of migratory birds in the Piaçabuçu Protection Area, Alagoas, Brazil. Every year, thousands of limicola and migratory birds winter on the South American coast, between September and April, where they acquire body mass and undergo moulting before returning to their reproduction sites. Qualitative and quantitative studies were performed in the Piaçabuçu Protection Area located in the state of Alagoas, Brazil, by direct counting, aiming to follow upon the birds' seasonal migration. Five species of Charadriidae family were recorded: Vanellus chilensis (Wagler, 1827); Pluvialis squatarola (Linnaeus, 1758); Charadrius semipalmatus Bonaparte, 1825; Charadrius collaris Vieillot, 1818 and Charadrius wilsonia (Ord, 1814), and five of the Scolopacidae family: Arenaria interpres (Linnaeus, 1758); Actitis macularius (Linnaeus, 1766); Catoptrophorus semipalmatus (Gmelin, 1789); Calidris pusilla (Linnaeus, 1766) and Calidris alba (Pallas, 1764). Pluvialis squatarola, Charadrius semipalmatus, Charadrius collaris, Arenaria interpres, Calidris pusilla and Calidris alba were considered constant (present in over 50\% of observation time). Charadrius semipalmatus and Calidris alba showed highest level of frequency during November and December and March and September, respectively. Spearman correlation shows a strong dependency on migration of this species. The birds' loyalty to the Piaçabuçu area seen in this research show that this is a preferred site for wintering thus reinforcing its importance for the conservation of the migratory species that utilize the site. KEY WORDS. Census; Charadriidae; Scolopacidae.
\end{abstract}

RESUMO. Todos os anos milhares de aves limícolas e migratórias invernam ao longo da costa da América do Sul, entre setembro e abril, onde adquirem massa corpórea e realizam mudas para retornar aos sítios de reprodução. Estudos quali-quantitativos foram realizados na Área de Proteção Ambiental de Piaçabuçu, Alagoas, Brasil, através da contagem direta, objetivando o acompanhamento das flutuações sazonais da avifauna migrante. Foram registradas cinco espécies da família Charadriidae: Vanellus chilensis (Wagler, 1827); Pluvialis squatarola (Linnaeus, 1758); Charadrius semipalmatus Bonaparte, 1825; Charadrius collaris Vieillot, 1818 e Charadrius wilsonia (Ord, 1814) e cinco espécies da família Scolopacidae: Arenaria interpres (Linnaeus, 1758); Actitis macularius (Linnaeus, 1766); Catoptrophorus semipalmatus (Gmelin, 1789); Calidris pusilla (Linnaeus, 1766) e Calidris alba (Pallas, 1764). Pluvialis squatarola, Charadrius semipalmatus, Charadrius collaris, Arenaria interpres, Calidris pusilla e Calidris alba foram consideradas constantes (presentes em mais de $50 \%$ das observações). Charadrius semipalmatus e Calidris alba apresentaram os maiores índices de freqüêencia de ocorrência nos meses de novembro e dezembro e, março e setembro, respectivamente. A correlação de Spearman demonstra uma forte dependência na migração destas espécies. A fidelidade dessas aves a APA de Piaçabuçu observada nessa pesquisa indica ser a área um sítio de invernada, reforçando sua importância para a conservação das espécies migratórias que utilizam o local.

PALAVRAS-CHAVE. Censo; Charadriidae; Scolopacidae.

Dentre as províncias biogeográficas, a região Neotropical apresenta maior biodiversidade, tanto em seus aspectos florísticos e faunísticos, quanto na sua multiplicidade de ambientes aquáticos (SICK 1997).

Todos os anos milhares de aves provenientes do Ártico migram para a América do Sul, devido à proximidade do outono boreal. Movimentam-se no sentido sul (Harrington et al. 1986), onde várias espécies invernam, principalmente nas costas marítimas do continente, no período de setembro a abril. Elas passam a maior parte do seu ciclo de vida no Continente Sul-Americano (ANTAS 1984).

O Brasil constitui um dos países que apresenta uma das

Revista Brasileira de Zoologia 23 (3): 865-869, setembro 2006 
maiores biodiversidades do planeta, estando esta riqueza biológica associada, sobretudo, à diversidade de ambientes aquáticos. Dentre os componentes faunísticos desses ecossistemas, SPAANs (1978) ressalta as aves paludínicas, continentais e as limícolas neárticas, com espécies residentes e migratórias.

Dentre os deslocamentos de aves que ocorrem no Brasil, destacam-se as migrações do inverno do Norte (inverno boreal) e do inverno do Sul (inverno austral). Quanto ao extremo Norte, são centenas e até milhares de indivíduos, que chegam e invernam, ou seja, utilizam estas áreas para pouso e alimentação, logo após o período reprodutivo, que ocorre de maio a julho (Sıck 1997). Ao longo da costa brasileira, desde o Amapá até o Rio Grande do Sul são encontrados vários sítios de invernada, sendo esses de extrema importância para a conservação e manutenção destas espécies, a exemplo da Ilha de Campechá e Lençóis Maranhenses (Maranhão), a Coroa do Avião (Pernambuco), a Lagoa do Peixe (Rio Grande do Sul) (Telino-JúnIor et al. 2003), e a Área de Proteção Ambiental de Piaçabuçu (Alagoas).

A caracterização da estrutura das comunidades de aves é utilizada pelos ornitólogos para comparar a avifauna de diferentes ecossistemas e/ou acompanhar as variações estacionais ou anuais de determinadas comunidades locais (LyrA-Neves et al. 2004).

Neste sentido o principal objetivo deste trabalho foi o levantamento e monitoramento das populações de aves migratórias que anualmente utilizam a Área de Proteção Ambiental de Piaçabuçu, registrar suas flutuações e mapear áreas de uso, através de censo direto, contribuindo assim, para subsidiar políticas de conservação desses recursos.

\section{MATERIAL E MÉTODOS}

\section{Área de estudo}

A Área de Proteção Ambiental de Piaçabuçu (APA Piaçabuçu) foi criada de 1983 com o objetivo de proteger as tartarugas marinhas e as aves migratórias que utilizam aquela área. De acordo com a Lei 9.985/00 do Sistema Nacional de Unidade de Conservação da Natureza (SNUC), trata-se de uma unidade de conservação pertencente ao Grupo de Uso Sustentável, localizada no extremo meridional do estado de Alagoas $\left(10^{\circ} 20^{\prime}\right.$ $\mathrm{S}$ e $36^{\circ} 20^{\prime} \mathrm{W}$ ) limitando-se ao Sul com o rio São Francisco, a Leste e a Norte com o Oceano Atlântico e a Oeste, com uma linha paralela à praia do Peba (MARra 1989) (Fig. 1).

A APA Piaçabuçu possui 18 mil hectares, com áreas alagadas, florestas de restinga e um mosaico de espécies vegetais e animais. Consiste em uma região caracterizada pela presença de dunas e gramíneas em expansão. As dunas são um cordão arenoso de 19 km que acompanha a praia desde o Pontal do Peba à foz do rio São Francisco, com a largura variando entre 700-800 m de largura, margeando o lado oposto ao continente. Segundo a classificação de Koppen, apresenta clima tropical chuvoso com verão seco, seguido por regime pluviométrico caracterizado por uma irregularidade tanto mensal quanto anual. O regime de ventos tem uma orientação E-SE no inverno e NE no verão (MARRA 1989).

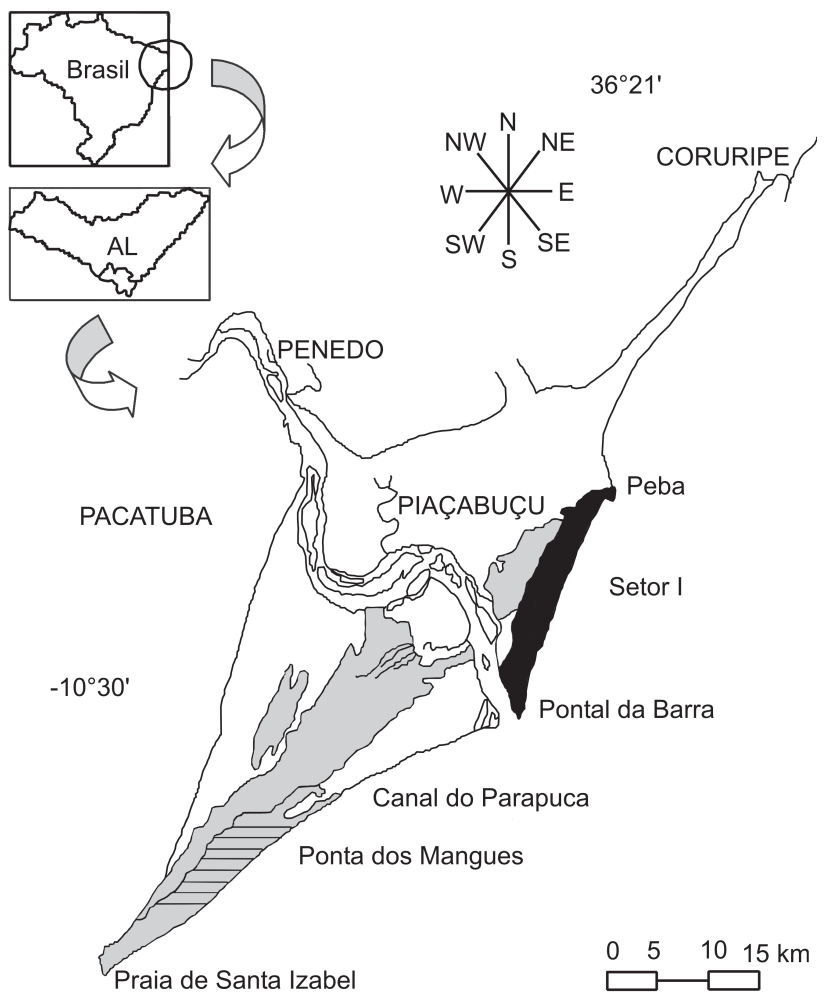

Figura 1. Mapa da Área de Proteção Ambiental de Piaçabuçu, Alagoas, representada no Setor I, incluindo as áreas do entorno, adaptado de Barbosa \& Domingues (2004).

MORRISON et al. (1989) citam que os componentes orgânicos dos sedimentos submersos são significativos, sobretudo, na foz do rio São Francisco, nos bancos de lama, e nas áreas atrás dos recifes e nas lagoas costeiras. Próximo ao povoado de Pontal do Peba encontra-se um pequeno manguezal. A presença de bancos de lama favorece a colonização dos camarões Penaeus schmitti Burkenroad, 1936 (camarão-branco) e Penaeus brasiliensis Latreille, 1817 (camarão-rosa), sendo, portanto, a pesca a principal atividade econômica da região.

\section{Métodos}

O trabalho foi realizado mensalmente entre junho de 2004 e setembro de 2005, durante três dias consecutivos. O método utilizado foi o de contagem direta, descrito por Вівву et al. (1992), em que o observador, em um ponto fixo, desenvolve uma contagem individual, com o auxílio de luneta ou binóculo. Para este estudo foi utilizado binóculo Samsung (10 X $25 \mathrm{~mm}$ ). Os censos foram realizados percorrendo-se a área com um veículo, devido à extensão da faixa de praia, obedecendose o regime de baixa-mar, entre o povoado de Pontal do Peba e a foz do rio São Francisco, totalizando um percurso de $18 \mathrm{~km}$. No percurso foram estabelecidos pontos fixos de observação obedecendo-se à concentração dos diversos bandos. 

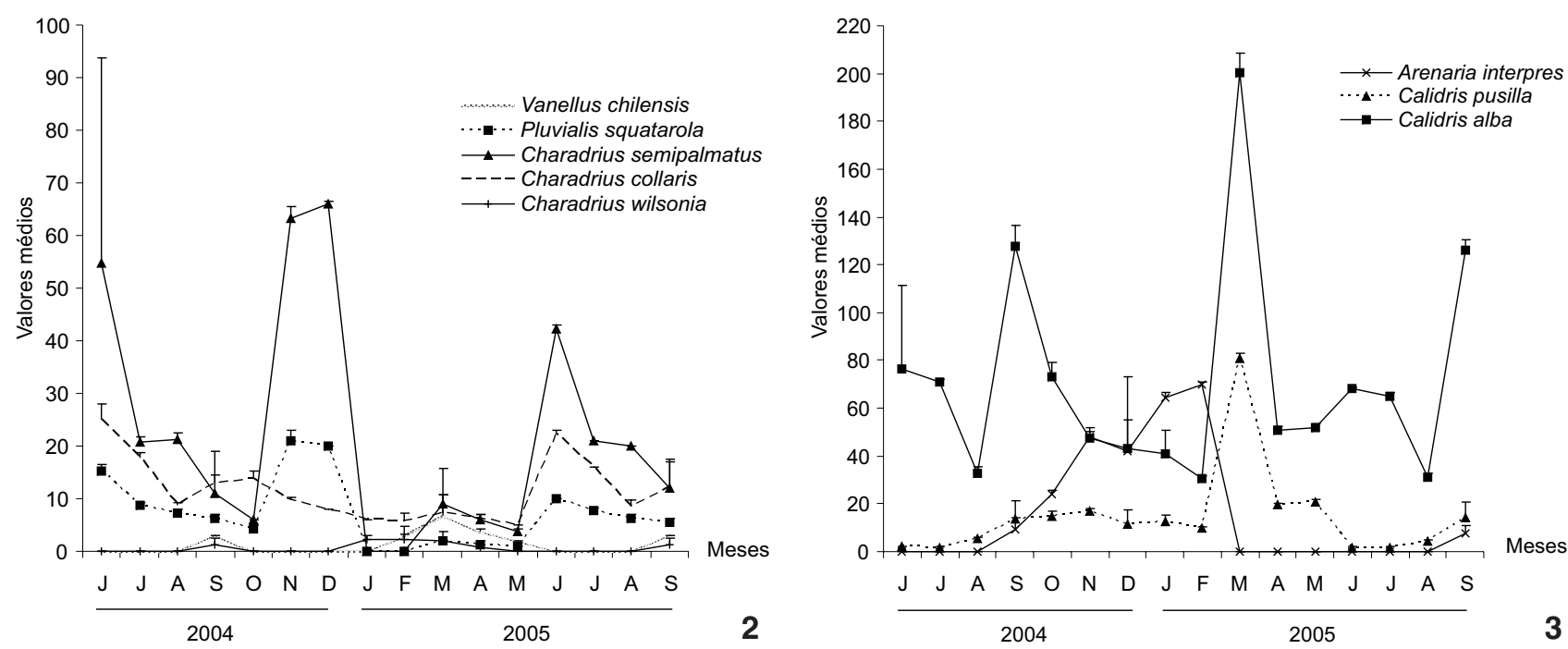

Figuras 2-3. Flutuação sazonal da freqüência média mensal das espécies realizada através de censo direto, na Área de Proteção Ambiental de Piaçabuçu, Aalagoas, entre junho de 2004 e setembro de 2005: (2) Vanellus chilensis (espécie residente), Pluvialis squatarola, Charadrius semipalmatus, Charadrius collaris (espécie residente) e Charadrius wilsonia; (3) Arenaria interpres, Calidris pusilla e Calidris alba.

Os indivíduos foram identificados segundo HarRISON (1983) e Hayman et al. (1986), a ordem filogenética segue Sick (1997).

Para a flutuação sazonal foi utilizada a média das contagens mensais, para aquelas espécies consideradas constantes, seguindo-se o estabelecido por Olmos \& SiLva (2002) que alegam ser esta metodologia a que possibilita uma estimativa mais precisa das populações que utilizam uma determinada área.

A similaridade entre as comunidades faunísticas foi obtida através do índice de Bray-Curtis, através da média ponderada das abundâncias mensais.

A constância foi expressa através do número de meses contendo a espécie, dividido pelo número total de meses de coleta (observação), através da equação C = p x 100/P, onde: p corresponde ao número de contagens contendo a espécie e $\mathrm{P}$ o número total de contagens, sendo: constante (quando presentes em mais de $50 \%$ das contagens), acessória (quando presente de $25 \%$ a $50 \%$ das contagens) e acidental (quando presente em menos de $25 \%$ das contagens) DAJOz (1983).

O coeficiente de correlação de Spearman foi desenvolvido através do programa Statistica versão 5.0 com um nível de significância em $\mathrm{p}<0,05$. Considerou-se Forte Correlação (FC) os valores obtidos entre 0,70 e 0,89 e Correlação Muito Forte (CMF) para valores entre 0,90 e 1,00 (Fowler \& COHEN 1988).

\section{RESULTADOS E DISCUSSÃO}

Foram registradas dez espécies, sendo cinco da família Charadriidae: Vanellus chilensis (Wagler, 1827), Pluvialis squatarola (Linnaeus, 1758), Charadrius semipalmatus Bonaparte, 1825, C. collaris Vieillot, 1818 e C. wilsonia (Ord, 1814) e cinco da família Scolopacidae: Arenaria interpres (Linnaeus, 1758), Actitis macularius (Linnaeus, 1766), Catoptrophorus semipalmatus (Gmelin,
1789), Calidris pusilla (Linnaeus, 1766) e C. alba (Pallas, 1764).

Foram consideradas espécies constantes Pluvialis squatarola, Charadrius semipalmatus, C. collaris, Arenaria interpres, Calidris pusilla e C. alba.

Charadrius semipalmatus e Calidris alba apresentaram os maiores índices de freqüência de ocorrência nos meses de novembro e dezembro e, setembro e março respectivamente, concordando parcialmente com Lyra-Neves et al. (2004) que citam também o mês de maio para Charadrius semipalmatus para a Coroa do Avião, Pernambuco.

$\mathrm{O}$ alto número de indivíduos de C. semipalmatus também no mês de junho, período em que esta espécie deveria estar em suas áreas de reprodução, é comentado por AzEvEDo-JúnIor \& LARRAZÁBAL (1994) e AzEVEDo-JúNIOR et al. (2001a, b). Estes autores referem-se à permanência de jovens, sub-adultos e adultos, que não concluem as mudas das penas de vôo em seu sítio de invernada, aguardando o próximo período reprodutivo para seguirem sua migração.

Segundo Myers et al. (1985a, b) as rotas migratórias das grandes populações de Calidris alba se dão pela costa do Pacífico e pelo Brasil Central, sendo a costa do Atlântico pouco utilizada. AzEvedo-Júnior et al. (2001, 2002), LARRAZÁBAL et al. (2002) e LyRANeves (2004) defendem a importância da rota do Atlântico para o monitoramento desta espécie, no nordeste do Brasil. TelinoJúnIOR et al. (2003) comentam ainda a alta freqüência de C. alba para a Coroa do Avião com bandos de mais de 500 indivíduos. Nesta pesquisa o alto percentual de registros de C. alba, corrobora com a importância dos sítios de invernada da costa Atlântica.

Em relação à flutuação, a partir das médias das contagens, as espécies foram agrupadas por família, Charadriidae e Scolopacidae respectivamente. Registraram-se picos em novem- 
bro para P. squatarola $(\mathrm{n}=21$; D.P. $=0,12)$, dezembro para $C$. semipalmatus $(\mathrm{n}=66$; D.P. $=0,39)$, junho para C. collaris $(\mathrm{n}=$ 25; D.P. $=0,03)$, fevereiro para A. interpres $(\mathrm{n}=70$; D.P. $=0,01)$ e março para C. pusilla $(\mathrm{n}=80,7$; D.P. $=0,57)$ e $C$. alba $(\mathrm{n}=$ 200,3; D.P. = 0,8) (Figs 2 e 3).

A correlação de Spearman demonstrou uma forte dependência na migração destas espécies. Nos meses de novembro e dezembro de 2004 e maio de 2005 foi observada uma forte correlação positiva entre $P$. squatarola e $C$. pusilla ( $\mathrm{rs}=0,893$ ) e uma correlação muito forte entre C. pusilla e C. alba $(\mathrm{rs}=0,912)$. De acordo com Morrison (1984) e LARrazábal et al. (2002) as aves neárticas se movimentam com a proximidade do inverno boreal.

Quanto à similaridade, foi constatado a formação de dois grandes grupos com espécies que se apresentaram semelhantes quanto ao período de suas maiores médias nas contagens realizadas (Fig. 4). Em relação aos meses de estudo obteve-se $\mathrm{R}_{\text {Global }}$ $=0,623(p=0,05)$ indicando diferenças altamente significativas entre os anos analisados quanto à composição da avifauna.

A fidelidade dessas aves a APA Piaçabuçu observada nessa pesquisa indica ser a área um sítio de invernada, reforçando sua importância para a conservação das espécies migratórias que utilizam o local, sendo estas fiéis aos seus sítios de invernada (Tab. I) em suas movimentações sazonais (CASTRo \& Myers 1987, AZEVEDO-JúNIOR et al. 2002).

Um monitoramento constante deve ser realizado anualmente para acompanhar a variação populacional dessas aves. Só assim será possível avaliar declínios e/ou aumentos populacionais ocorridos ao longo dos anos e inferir qual a decorrência deste fenômeno (Telino-Júnior et al. 2003). Segundo Cordeiro et al. (1996) a conservação de aves migratórias está diretamente rela-

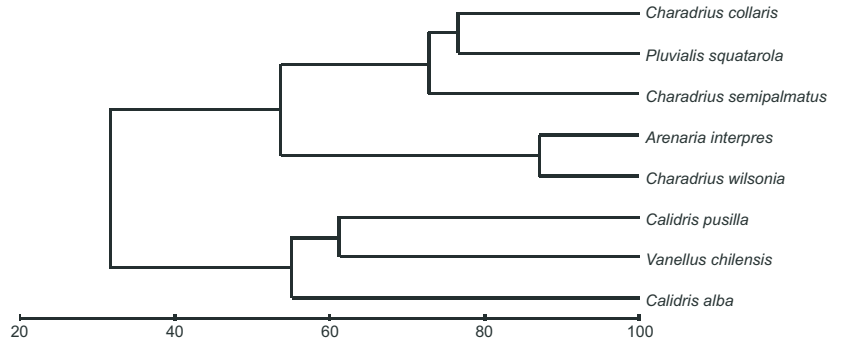

Figura 4. Dendrograma de similaridade entre as comunidades faunísticas na Área de Proteção Ambiental de Piaçabuçu, Alagoas.

cionada com a identificação de sítios de alimentação, repouso e reprodução, e que a perda de um sítio pode acarretar na diminuição e até mesmo na extinção de alguma espécie. Ainda defende que trabalhos de monitoramento de populações migratórias são fundamentais para conservação das mesmas.

\section{AGRADECIMENTOS}

Às Universidades Federal de Pernambuco (UFPE) e Federal Rural de Pernambuco (UFRPE), à Fundação Coordenação de Aperfeiçoamento de Pessoal de Ensino Superior (CAPES) ao Instituto Brasileiro do Meio Ambiente e dos Recursos Naturais Renováveis (IBAMA/AL) e ao Mestrado em Biologia Animal, UFPE, pelo suporte concedido.

\section{REFERÊNCIAS BIBLIOGRÁFICAS}

ANTAS, P.T.Z. 1984. Migration of neartic shorebirds (Charadriidae and Scolopacidae) in Brasil - flyways and thair different seasonal use. Wader Studie Group Bulletin, Tring, 39: 52-56.

Tabela I. Comparação de dados de constância de aves migratórias na Coroa do Avião, Pernambuco (TeLINO-JúNIOR et al. 2003), com os resultados obtidos nos censos realizados na Área de Proteção Ambiental de Piaçabuçu, Alagoas, entre junho de 2004 e setembro de 2005.

\begin{tabular}{|c|c|c|c|c|}
\hline \multirow{2}{*}{ Espécies } & \multicolumn{2}{|c|}{ Agosto/1996 a julho/1997 } & \multicolumn{2}{|c|}{ Junho/2004 a setembro/2005 } \\
\hline & Presença (\%) & Status & Presença (\%) & Status \\
\hline Vanelus chilensis & 0,0 & Ausente & 30 & Acessória \\
\hline Pluvialis squatarola & 100,0 & Constante & 70 & Constante \\
\hline Charadrius semipalmatus & 83,3 & Constante & 80 & Constante \\
\hline Charadrius collaris & 67,0 & Constante & 100 & Constante \\
\hline Charadrius wilsonia & 8,0 & Acidental & 40 & Acessória \\
\hline Avenaria interpres & 100,0 & Constante & 60 & Constante \\
\hline Actitis macularius & 0,0 & Ausente & 10 & Acidental \\
\hline Catoptrophorus semipalmatus & 0,0 & Ausente & 20 & Acidental \\
\hline Calidris fuscicollis & 8,0 & Acidental & 0 & Ausente \\
\hline Calidris pusilla & 75,0 & Constante & 100 & Constante \\
\hline Calidris Alba & 100,0 & Constante & 100 & Constante \\
\hline Numenius phaeopus & 8,3 & Acidental & 0 & Ausente \\
\hline Sterna hirundo & 43,8 & Acessória & 0 & Ausente \\
\hline Thalasseus sandicensis & 32,0 & Acessória & 0 & Ausente \\
\hline
\end{tabular}

Revista Brasileira de Zoologia 23 (3): 865-869, setembro 2006 
Azevedo Júnior, S.M. DE \& M.E.L. LarrazÁbal. 1994. Censo de aves limícolas na Coroa do Avião, Pernambuco, Brasil, informações de 1991 a 1992. Revista Nordestina de Zoologia, Recife, 1 (1): 263-277.

Azevedo-Júnior, S.M. de.; M.M. Dias-Filho \& M.E. de Larrazábal. 2001a. Plumagem e mudas de Caradriiformes (Aves) no litoral de Pernambuco, Brasil. Revista Brasileira de Zoologia, Curitiba, 18 (3): 657-672.

Azevedo-Júnior, S.M. DE.; M.M. Dias-Filho; M.E. de Larrazábal; W.R. Telino-Júnior; R.M. Lyra-Neves \& C.J.G. Fernandes. 2001b. Recapturas e recuperações de aves migratórias no litoral de Pernambuco, Brasil. Ararajuba, Brasília, 9 (1): 33-42.

Azevedo-Júnior, S.M. De.; M.M. Dias-Filho; M.E. Larrazábal \& C.J.G. Fernandes. 2002. Capacidade de vôo de quatro espécies de Chadriformes (Aves) capturadas em Pernambuco, Brasil. Revista Brasileira de Zoologia, Curitiba, 19 (1): 183-189.

Barbosa, L.M. \& J.M.L. Dominguez. 2004. Coastal dune fields at the São Francisco river strandplain, northeastern Brazil: morphology and environmental controls. Earth Surface Processes and Landforms, Leeds, 29 (4): 443-456.

Bibby, C.J.; N.D. Burgess \& D.A. Hill. 1992. Bird censes techniques. London, Academic Press, 257p.

Castro, G. \& J.P. Myers. 1988. A statistical method to estimate the cost of flight in birds. Journal of Field Ornithology, Lawrence, 59 (4): 369-380.

Cordeiro, P.H.C.; J.M. Flores \& J.L.X. do Nascimento. 1996. Análise das recuperações de Sterna hirundo no Brasil entre 1980 e 1994. Ararajuba, Belo Horizonte, 4 (1): 3-7.

DAJOz, R. 1983. Ecologia geral. As características das populações animais. Petrópolis, Vozes, $3^{\text {rd }}$ ed., IV+474p.

Fowler, J. \& L. COHEN. 1988. Statistics for ornithologists. Leicester, British Trust for Ornithology, II+150p.

Harrington, B.; P.T.Z. Antas \& F. Silva. 1986. Observations of Common Terns in South Brazil. Journal of Field Ornithology, Lawrence, 57: 222-224.

HARRISOM, P. 1983. Seabirds: an identification guide. Boston, Houghton Mifflin Company, 448p.

Hayman, P.; J. Marchant \& T. Prater. 1986. Shorebird. An identification guide to the waders of the world. London, Groom Helm, 412p.

Larrazabal, M.E. de; S.M. de Azevedo-Júnior \& O. Pena. 2002. Monitoramento de aves limícolas na Salina Diamante Branco, Galinhos, Rio Grande do Norte, Brasil. Revista Brasileira de Zoologia, Curitiba, 19 (4): 1081-1089.

Lyra-Neves, R.M.; S.M. De.Azevedo-JúnIOR \& W.R. Telino-JúnIor. 2004 Monitoramento do maçarico-branco, Calidris alba (Pallas) (Aves, Scolopacidae), através de recuperações de anilhas coloridas, na Coroa do Avião, Igarassu, Pernambuco, Brasil. Revista Brasileira de Zoologia, Curitiba, 21 (2): 319-324.

Marra, R.J.C. 1989. Educação Ambiental Preliminar a uma Unidade de Conservação. O caso da APA de Piaçabuçú/ Estação Ecológica Praia do Peba/AL. Brasília, Ibama, 346p. Morrison, R.I.G. 1984. Migration systems of some new world shorebirds, p. 125-202. In: J. Burger \& B.L. Olla (Eds). Shorebirds: migration and foraging behavior. New York, Plenum Press, Behavior of marine animals, vol. 6, 344p.

Morrison, R.I.G.; R.K. Ross \& P.T.Z. Antas. 1989. Padrões gerais de distribuição de aves litorâneas neárticas na América do Sul, p. 179-210. In: Atlas of neartic shorebirds on the coast of South America. Ottawa, Canadian Wildlife Service, vol. 2, 344p.

Morrison, R.I.G.; R.K. Ross \& P.T.Z. Antas. 1989. Brazil, p. 178211. In: R.I.G. Morrison \& R.K. Ross (Eds). Atlas of Neartic shorebirds on the coast of South America. Ottawa, Canadian Wildlife Service, vol. 2, 325p.

Myers, J.P.; J. Maron \& M. Sallaberry. 1985a. Going to the extremes: why do sanderlings migrate to the neotropics. Neotropical Ornithology, Ornithological Monographs, St. Louis, 36: 520-535.

Myers, J.P.; M. Sallaberry; G. Castro; J.L. Maron; E. Ortiz; C.T. Schick \& E. Tabilo. 1985b. Migración interhemisférica del playero blanco (Calidris alba): nuevas observaciones del Programa Panamericano de Chorlos e Playeros. El Volante Migratorio, Lima, 4: 23-27.

Olmos, F. \& R. Silva E Silva. 2002. The avifauna of a southeastern Brazilian mangrove swamp. International Journal of Ornithology, Belo Horizonte, 4 (3/4): 115-207.

SICK, H. 1997. Ornitologia brasileira. Rio de Janeiro, Editora Nova Fronteira, 912p.

SPAANS, A.L. 1978. Status and numerical fluctuations of some North American waders along the Surinam Coast. Wilson Bulletin, Lawrence, 90 (1): 60-83.

Telino-Júnior, W.R.; S.M. De. Azevedo-Júnior. \& R.M. de. L. Neves. 2003. Censo de aves migratórias (Charadriidae, Scolopacidae e Laridae) na Coroa do Avião, Igarassu, Pernambuco, Brasil. Revista Brasileira de Zoologia, Curitiba, 20 (3): 451-456.

Recebido em 17.I.2006; aceito em 29.VIII.2006. 